\title{
Primera jornada de detección precoz del cáncer de piel, Asocolderma 2011, reporte de la experiencia en Medellín, Colombia
}

\author{
Margarita María Velásquez ${ }^{1}$, Ángela Zuluaga de Cadena ${ }^{2}$ \\ 1. Médica dermatóloga, Doctora en Ciencias Básicas Biomédicas - Inmunología; jefe, Sección de Dermatología, Universidad de Antioquia, Medellín, \\ Colombia; Centro de Investigaciones Dermatológicas, CIDERM; miembro de número de ASOCOLDERMA \\ 2. Médica dermatóloga; miembro honorario y presidente honorario de la actual Junta Directiva de Asocolderma
}

\section{Resumen}

Con el propósito de proyectar la Dermatología a la población general, conocer algunos aspectos del problema del cáncer de piel e iniciar campañas masivas de educación nacional, Asocolderma inició este programa en Medellín como ciudad piloto.

Se presentan los resultados de la primera jornada realizada del 17 al 19 de mayo de 2011, con la participación de las escuelas de dermatología del CES y la Universidad de Antioquia, y otros dermatólogos de la ciudad. De las 2.404 solicitudes telefónicas, se adjudicaron 1.265 citas. A 246 pacientes se les sospecharon lesiones premalignas o malignas, a 149 se les tomó biopsia y a los demás se les sospechó clínicamente queratosis actínicas o nevus atípicos. Se detectaron dos pacientes con melanoma maligno, 14 con carcinoma escamocelular, 59 con carcinoma basocelular y 1 con porocarcinoma. Se obtuvo información importante sobre las prácticas de exposición y protección solar en este grupo de pacientes.

Palabras Clave: jornada de detección de cáncer de piel, educación, fotoprotección, melanoma maligno, carcinoma escamocelular, carcinoma basocelular.

\section{Summary}

In order to project the Dermatology to the general population, to learn about some aspects of the problem of skin cancer and to initiate national public education campaigns, Asocolderma started this program in Medellin as a pilot city. We present the results of the first program realized on May 17, 18 and 19, 2011, with the participation of Universidad CES and Universidad de Antioquia Dermatology grous, and other dermatologists in the city.

Of the 2,404 telephone requests, 1,265 citations were awarded. Malignant or premalignant lesions were suspected in 246 patients, 149 biopsies were taken and atypical nevi or actinic keratoses were suggested clinically in the remainder. There were 2 patients with malignant melanoma, 14 squamous cell carcinomas, 59 basal cell carcinomas, and 1 porocarcinoma. Important information was obtained on the practices of sun exposure and protection in this patient group.

KEYWORDS: Program of skin cancer screening, education, photoprotection, malignant melanoma, squamous cell carcinoma, basal cell carcinoma.

\section{Correspondencia:}

Margarita María Velásquez

Email: mmvelasquez@yahoo.com

Recibido: 20 de marzo de 2012. Aceptado: 25 abril de 2012.

\section{Conflictos de intereses:}

Los Laboratorios Percos contribuyeron con Asocolderma en la financiación y apoyo logístico de la jornada de cáncer de piel, pero no participaron en el análisis de los datos ni en la elaboración del manuscrito.

Asocolderma les encargó el análisis de los datos directamente a Margarita Velásquez y Ângela Zuluaga. Las autoras no recibieron ningún tipo de retribución económica ni contraprestación. 


\section{Introducción}

Debido al cambio climático global y a la exposición solar, tanto ocupacional como recreativa, el cáncer de piel melanoma y no melanoma está en aumento, lo que hace necesaria la implementación de campañas educativas a la población general sobre los hábitos de vida saludable, el uso de las medidas de protección solar, el autoexamen de piel y la oportuna consulta al dermatólogo'.

Las medidas de prevención primaria incluyen el evitar la exposición solar en la horas de mayor irradiación ultravioleta, el uso de sombrero, gafas y manga larga, y la aplicación adecuada del protector solar. Las medidas de prevención secundaria están dirigidas a la población por medio de campañas educativas y de detección precoz ${ }^{2}$.

Uno de los factores más importantes en la génesis del melanoma maligno, el carcinoma basocelular y el carcinoma escamocelular, es la exposición acumulada o crónica a la radiación ultravioleta, lo que se suma a la posible presencia de mutaciones genéticas ${ }^{3}$. Todos los esfuerzos encaminados a la educación y a la detección precoz están plenamente justificados.

El melanoma maligno es un tumor derivado de los melanocitos, su comportamiento biológico varía de acuerdo con la respuesta inmunológica del individuo y, sin tratamiento, genera metástasis a ganglios linfáticos y a otros órganos, siendo más frecuente el compromiso pulmonar, hepático y cerebral.

El melanoma maligno corresponde a $3 \%$ de los cánceres cutáneos, pero produce el $65 \%$ de las muertes por cáncer de piel. El reconocimiento y el tratamiento precoces son muy importantes porque la supervivencia a 10 años es mayor de $90 \%$ cuando se detecta tempranamente (menor de $1 \mathrm{~mm}$ en la escala de Breslow). Entre los factores que predisponen a esta neoplasia se encuentran el antecedente de insolaciones en la infancia, la presencia de más de 25 nevus según algunos autores o más de 50 nevus según otros, el síndrome de nevus displásicos, el xeroderma pigmentoso, y el antecedente personal y familiar de melanoma, entre otros ${ }^{4-6}$. A pesar de lo delicado de este tipo de cáncer y de que es visible a la inspección clínica, es poco el conocimiento que tiene la población general de los signos de alarma. Para el año 2000, la American Academy of Dermatology reveló que la mayoría de los estadounidenses desconocían los signos de alarma del melanoma y menos de la mitad se había practicado un examen de la piel.

La primera campaña de detección masiva se llevó a cabo en Australia en 1963-1968, dentro del Queensland Melanoma Project ${ }^{8}$. En los años ochenta, en Estados Unidos se creó el American Academy of Dermatology National Melanoma Skin Cancer Screening Program, que tuvo lugar en la primavera de cada año, en el que se revisaron más de 750.000 personas en el período 19851995 y, 282.535, en 1992-1994'. El mayor desarrollo de las campañas de detección masiva tuvo lugar en los años noventa en Holanda, Alemania y Estados Unidos. Por el éxito de estas campañas, la American Academy of Dermatology determinó que cada primer lunes de mayo se denominara Monday Melanoma Day y que, además, el mes de mayo se dedicara al cáncer cutáneo. Siguiendo esta iniciativa, en el año 200o, la European Academy of Dermatology and Venereology instauró una campaña de detección precoz de melanoma denominada Euromelanoma Day, que se realiza en diferentes países como España, Bélgica, Italia, Francia, República Checa, Grecia, Dinamarca, Finlandia, Islandia, Malta, Portugal, Eslovaquia, Suecia y el Reino Unido ${ }^{10}$.

En los estudios de la American Academy of Dermatology basados en los datos de las campañas de detección de cáncer de piel, se han identificado poblaciones de alto riesgo por criterios que se resumen en el acrónimo HARMM (History, Age, Regular dermatologist absent, Mole changing, Male), es decir, antecedentes personales de melanoma, edad mayor de 50 años, ausencia de valoración dermatológica, cambios en las lesiones y sexo masculino. Los individuos de alto riesgo (cuatro a cinco factores), corresponden a 5,8 \% del total de los evaluados en las campañas y a 13,6 \% de los pacientes con lesiones sugestivas de melanoma maligno. El riesgo relativo de detectar lesiones sugestivas de melanoma maligno es 4,4 veces mayor para la población de alto riesgo que para la de bajo riesgo ${ }^{3}$.

El cáncer de piel no melanoma incluye al carcinoma escamocelular y al basocelular. El principal factor de riesgo es la exposición solar, que ha llevado a un aumento de los casos en los últimos 30 años. El incremento es más notorio para el carcinoma escamocelular que para el basocelular y se ha descrito que en la patogénesis del basocelular juegan un papel más importante las exposiciones intensas ocasionales. Entre los factores asociados al cáncer no melanoma múltiple, se han descrito la edad, la presencia de más de seis nevus en el antebrazo, la incapacidad para broncearse, la historia familiar de melanoma y el antecedente de más de 10 quemaduras solares ${ }^{11}$.

El carcinoma basocelular representa el $70 \%$ de los cánceres de piel no melanoma. La mayoría de los estudios habían reportado una proporción de basocelular:escamocelular de 4:1, sin embargo, el notorio aumento del carcinoma escamocelular ha llevado a proporciones de 1:1 en diferentes poblaciones, lo que refleja un aumento desproporcionado del escamocelular con el aumento de la exposición solar. En general, el pronóstico es bueno, la presencia de metástasis es extremadamente rara, excepto en los casos de diagnóstico 
tardío o en formas agresivas o de difícil manejo, como el carcinoma basocelular morfeiforme ${ }^{12}$.

En cuanto al carcinoma escamocelular, el principal factor ambiental para su desarrollo y para la aparición de lesiones precursoras como las queratosis actínicas y la queilitis actínica, entre otras, es la exposición solar. Su frecuencia está asociada a la dosis total acumulativa de radiación ultravioleta. Tanto la radiación ultravioleta B (UVB) como la radiación ultravioleta A (UVA) están relacionadas con la carcinogénesis. En la mayoría de casos, la radiación ultravioleta induce mutaciones en el gen $p 53$. El carcinoma escamocelular es más frecuente en piel expuesta al sol, en individuos que trabajan al aire libre y en quienes usan cámaras bronceadoras. Las áreas anatómicas más afectadas son la cara, la espalda, las manos y los antebrazos. Entre los factores constitucionales, el más importante es el fototipo; este tipo de carcinoma es más frecuente en individuos con poca capacidad de broncearse (fototipos I, II y III de Fitzpatrick). Otros factores de riesgo son la infección por el virus del papiloma humano (HPV), especialmente en lesiones genitales y en inmunosuprimidos ${ }^{13-16}$.

La prevalencia publicada de queratosis actínicas en adultos mayores de 40 años varía entre 11 y $25 \%$ en el hemisferio norte y entre 40 y $60 \%$ en el hemisferio sur. Sin tratamiento, las queratosis actínicas pueden permanecer sin cambios, regresar espontáneamente o progresar a carcinoma escamocelular. La probabilidad de regresión espontánea en un año es de 15 a $25 \%$. Con modelos matemáticos se ha calculado que el riesgo de desarrollar carcinoma escamocelular a 10 años en individuos que tienen 7,7 queratosis actínicas es de $10 \%$. Según varios estudios, 5 a $20 \%$ de las queratosis actínicas progresan a carcinoma escamocelular en 10 a 25 años ${ }^{16,17}$.

El pronóstico de la mayoría de casos de carcinoma escamocelular es bueno, el riesgo de metástasis para las lesiones en áreas fotoexpuestas es de 2,3\% a cinco años y de $5,2 \%$ en seguimientos de más de cinco años. Las recaídas, las metástasis y la mortalidad asociadas a este carcinoma, generalmente, se asocian con un diagnóstico tardío o con variantes agresivas ${ }^{16,18}$.

En Colombia, específicamente en Medellín y el oriente antioqueño, se ha detectado que el índice de irradiación ultravioleta es mayor de 9 (promedio de 10). Esto demuestra que nuestra población está expuesta a niveles muy altos de radiación ultravioleta durante todo el año, por lo que se deben programar campañas en los medios de comunicación locales para advertir al público sobre los peligros de la exposición no controlada a la radiación ultravioleta ${ }^{19}$.

Aunque en Colombia se han realizado algunos esfuerzos educativos sobre la prevención del cáncer de piel, no se cuenta con programas establecidos y continuados para la educación y el diagnóstico masivo del cáncer de piel. Teniendo en cuenta lo anterior, la Asociación Colombiana de Dermatología, Asocolderma, inició las jornadas de detección del cáncer de piel, comenzando en Medellín a manera de ciudad piloto, para posteriormente ampliar el programa a otras ciudades del país.

Con el propósito de proyectar la dermatología a la población general, conocer algunos aspectos del problema del cáncer de piel e iniciar una campaña masiva de educación nacional, se planeó un estudio piloto en Medellín.

Se presentan los resultados de la primera jornada de detección precoz del cáncer de piel en Medellín, llevada a cabo los días 17, 18 y 19 de mayo de 2011, con la participación de las escuelas de dermatología de la Universidad CES y de la Universidad de Antioquia, y otros dermatólogos de la ciudad, con el apoyo del estudio histopatológico por parte de los profesores de Dermatopatología de la Universidad CES y la Universidad de Antioquia.

\section{Metodología}

Asocolderma, mediante la Asociación Antioqueña de Dermatología (SADE), convocó a los dermatólogos de Medellín para realizar la primera jornada de detección del cáncer de piel, iniciándola allí como ciudad piloto y con el propósito de implementar este programa en otras ciudades del país. Por medio de comunicación escrita, los dermatólogos participantes manifestaron el horario de su disponibilidad y compromiso. En marzo de 2011, se citó a los dermatólogos y se les motivó para donar un tiempo de consulta para la jornada del 17, 18 y 19 de mayo del mismo año. En total, 64 dermatólogos graduados y residentes de Dermatología de la Universidad de Antioquia y la Universidad CES participaron en la campaña de manera voluntaria y gratuita. $\mathrm{Al}$ mismo tiempo, se inició la difusión de la campaña por medios masivos de comunicación, y con la distribución de afiches y plegables educativos con los signos de alarma de las lesiones pigmentarias, de acuerdo con su ABCDE: asimetría, bordes irregulares, color variado, diámetro mayor de o,6 mm y evolución.

Los pacientes se seleccionaron y programaron por medio de una solicitud a una central telefónica, en la que se les hizo una primera encuesta para determinar el motivo de consulta y algunos factores asociados con el cáncer de piel. La central telefónica fue atendida por personal entrenado por dermatólogos. El número de pacientes por evaluar se calculó con base en el tiempo disponible de atención dermatológica. Los criterios de inclusión para asignar la cita fueron: personas de 18 años o mayores, sin importar el sexo, que manifestaran tener lesiones sospechosas de ser premalignas o malignas, 
detectadas en la encuesta telefónica prediseñada por dermatólogos.

Unos días antes de la jornada, los dermatólogos y las escuelas de Dermatología recibieron la lista de los pacientes asignados, seis por hora, y la papelería, incluyendo el formato de consentimiento informado y de evaluación clínica por el dermatólogo.

Los dermatólogos participantes evaluaron los pacientes asignados y, a los que tenían impresión clínica de cáncer o precáncer de piel, les diligenciaron el formato de evaluación por dermatólogo (segunda encuesta).

Ante la sospecha de este tipo de lesiones se tomaron biopsias de piel, previo consentimiento informado por escrito. Las biopsias fueron analizadas por profesores de Dermatopatología de la Universidad CES y la Universidad de Antioquia. Los reportes de biopsia fueron entregados a todos los pacientes, con las recomendaciones para continuar con su atención médica en su respectivo seguro de salud. Se envió una copia del reporte de la biopsia al dermatólogo que atendió al paciente y a la Entidad Promotora de Salud.

Los datos de la encuesta diligenciada en la central de llamadas para todos los pacientes que solicitaron cita y el formato de evaluación por el dermatólogo para los pacientes con sospecha lesiones malignas y premalignas, fueron tabuladas en una base de datos en Excel $\AA$ y analizadas con SPSS $®$, versión 15, con la asesoría de un experto en epidemiología.

Se hicieron los siguientes análisis: para el análisis univariado, se calcularon frecuencias y porcentajes para todas las variables del estudio; para el análisis bivariado, se cruzaron los diagnósticos clínicos con los histológicos, y se calcularon valores de sensibilidad y valor pronóstico positivo (VPP); para el cálculo de los porcentajes, no se tuvieron en cuenta los registros que estaban vacíos, es decir, se hizo solo sobre las respuestas obtenidas. El promedio de datos completos fue de $80 \%$.

\section{Resultados}

\section{Descripción de la población}

En la central telefónica se recibieron 2.404 solicitudes, de las cuales se adjudicaron 1.265 citas (52,6 \% del total de llamadas), para las personas que cumplieron los criterios de inclusión. Del total de pacientes que fueron citados, 856 fueron mujeres $(67,7 \%)$ y 409 hombres, la edad fue $48 \pm 16$ años (media \pm DE), 1.012 (80 \%) tenían seguro de salud (EPS o Sisbén). En la historia de exposición solar, el $33 \%(n=418)$ desarrollaba trabajo al aire libre y solo la mitad refería el uso de protector antisolar (50,9 \%); el 52,8 \% había tenido insolaciones en la in- fancia. Una tercera parte $(31,2 \%)$ tenía antecedentes personales o familiares de cáncer de piel.

Respecto a la aparición de cambios en la piel, dos terceras partes $(65,4 \%)$ se quemaban con facilidad, al $68,1 \%$ le salían "lunares" regularmente y el 57,3 \% tenía "lunares o granos" recientes, 29,9\% "pecas" y el 64,9\% manchas. Cuatrocientos ochenta $(33,2 \%)$ dijeron tener lunares en ojos o palmas, $793(62,7 \%)$, lunares de diferentes tonos de café y, 78 (6,2\%), azulosos.

Se encontró que las quejas más frecuentes fueron "manchas, pecas o lunares", en $72,6 \%$ de las personas (TABLA 1).

\section{Pacientes con sospecha de lesiones premalignas y malignas}

De los 1.265 pacientes citados para ser evaluados por los dermatólogos, 246 (19,4\%) presentaban sospecha de lesiones malignas y premalignas, y a estos se les analizaron los datos de la segunda encuesta (evaluación por el dermatólogo). A 149 (11,7 \%) se les tomó biopsia, a 55 pacientes se les hizo el diagnóstico clínico de queratosis actínicas, 39 de nevus atípicos y 3 con ambos diagnósticos (Tabla 2).

\begin{tabular}{lcc}
\hline \multicolumn{1}{c}{ Motivo de consulta } & $\mathbf{n}$ & $\%$ \\
\hline \hline Manchas, pecas o lunares & 919 & 72,6 \\
Grano, cáncer, lesión, herida, masa, espini- & 150 & 11,9 \\
lla, quemadura o costra & & \\
Prevención, revisión, diagnóstico & 87 & 6,9 \\
Antecedentes de cáncer (personal o familiar) & 49 & 3,9 \\
Otros & 35 & 2,8 \\
Sin dato & 25 & 2 \\
\hline \hline ToTAL & 1.265 & 100 \\
\hline \hline
\end{tabular}

TABLA 1. Motivos de consulta referidos por los pacientes en la entrevista telefónica.

\begin{tabular}{lcc}
\hline \hline & $\mathbf{n}$ & $\%$ \\
\hline \hline Con biopsia & 149 & 60,6 \\
Con queratosis actínicas sin biopsia & 55 & 22,4 \\
Con nevus atípico sin biopsia & 39 & 15,9 \\
Con nevus atípico y queratosis actínica sin & 3 & 1,2 \\
biopsia & & \\
\hline TotaL & 246 & 100 \\
\hline \hline
\end{tabular}

TABLA 2. Pacientes con sospecha de lesiones premalignas o malignas. 


\begin{tabular}{lccc}
\hline \multicolumn{1}{c}{ Variable } & Categoría & $\mathbf{n}$ & $\%$ \\
\hline Se protege con bloqueador solar & Sí & 126 & 51,2 \\
& No & 120 & 48,8 \\
Se quema con facilidad & Sí & 172 & 69,9 \\
Practica actividades al sol & No & 73 & 29,7 \\
& Sí & 114 & 54,0 \\
Se quemó al sol antes de los 18 años & No & 97 & 46,0 \\
& Sí & 111 & 53,1 \\
Trabaja o ha trabajado al aire libre & No & 98 & 46,9 \\
& Sí & 72 & 35,0 \\
Ha vivido en el campo & No & 134 & 65,0 \\
Toma sesiones de cámara bronceadora & Sí & 89 & 43,0 \\
& No & 118 & 57,0 \\
\hline \hline
\end{tabular}

\begin{tabular}{lccc}
\hline Variable & Categoría & $\mathbf{n}$ & $\%$ \\
\hline Estimación del número de nevus en el cuerpo & $<25$ & 125 & 68,7 \\
& $25-100$ & 49 & 26,9 \\
Presencia de nevus de unión & $>100$ & 8 & 4,4 \\
Presencia de nevus atípicos & Sí & 137 & 69,9 \\
& No & 59 & 30,1 \\
& Sí & 75 & 37,7 \\
& No & 124 & 62,3 \\
\hline
\end{tabular}

TABLA 3. Exposición a radiación ultravioleta de los pacientes con sospecha clínica de lesiones premalignas o malignas.
Tabla 4. Frecuencia de nevus en la población con sospecha de lesiones premalignas y malignas.
En cuanto a la exposición a radiación ultravioleta, el $53,1 \%$ había tenido quemaduras solares antes de los 18 años, el $54 \%$ practicaba actividades al sol, $35 \%$ trabajaba o había trabajado al aire libre, $43 \%$ había vivido en el campo y $12 \%$ había usado cámaras bronceadoras. Solo el 51,2 \% se protegía con bloqueador solar (TABLA 3).

Al evaluar los antecedentes de cáncer de piel, 12 (7,5\%) tenían antecedentes familiares de melanoma y, en los antecedentes personales, 1 había tenido melanoma maligno; 5, carcinoma escamocelular; 30, carcinoma basocelular, y 5, otros carcinomas de piel no melanoma.

En el examen físico de los pacientes a quienes se les consignó el dato del fototipo, la mayoría tenía fototipos ll y lll, $77(36,5 \%)$ y $83(39,3 \%)$, respectivamente, seguidos por $5(11,8 \%)$ con fototipo I, $23(10,9 \%)$ con el IV y $3(1,4 \%)$ con el V. Ninguno era de fototipo VI.

En total, 187 pacientes tenían nevus de cualquier tipo: en $68,7 \%$ eran menos de 25 nevus, en $26,9 \%$, de 25 a 100 nevus y, en el 4,4\%, más de 100; 137 de los pacientes tenían nevus de unión (69,9\%). A 75 personas se les hizo diagnóstico clínico de nevus atípico (37,7 \%) (Tabla 4).

La presencia de efélides se detectó en $49 \%$ de los pacientes y, de lentigos solares, en $75 \%$, lo que está asociado a la exposición solar crónica.
A 149 personas se les tomó biopsia, 87 mujeres $(58,4 \%)$ y 62 hombres 41,6\%), con edad promedio de 54 años (media de $54 \pm 16$ de desviación estándar), con un rango de 18 a 90 años. Se tomó una sola muestra a 123 pacientes, dos muestras a 19, tres biopsias a 4, y cuatro biopsias a 3 , para un total de 185 análisis histopatológicos.

Los diagnósticos clínicos con los que el dermatólogo remitió el espécimen de biopsia para estudio, fueron: melanoma maligno (21), carcinoma escamocelular (17), carcinoma basocelular (83), nevus atípicos (32), queratosis actínica (16), otros nevus (7), otros diagnósticos no malignos (7) y linfoma cutáneo (2). El estudio histológico arrojó los siguientes resultados: melanoma maligno (2), carcinoma escamocelular (14), carcinoma basocelular (59), nevus atípico (15), queratosis actínica (26), otros nevus (34), otros diagnósticos no malignos (34) y porocarcinoma (1) (TABLA 5).

La sensibilidad del diagnóstico clínico en relación con el resultado de histopatología como método de referencia, fue la siguiente: melanoma maligno, $100 \%$, carcinoma escamocelular, 42,9\%; carcinoma basocelular, 86,4 \%; nevus atípicos, 73,3 \%; queratosis actínica, 42,31 \%, otros nevus, $86,7 \%$, y otras lesiones no malignas 20,6\%. En la Tabla 6 se presentan los datos de 


\begin{tabular}{lcccc}
\hline \multirow{2}{*}{ Diagnóstico } & \multicolumn{2}{c}{ Clínico } & \multicolumn{2}{c}{ Histológico } \\
& $\mathbf{n}$ & \% & $\mathbf{n}$ & \% \\
\hline \hline Melanoma maligno & 21 & 11,4 & 2 & 1,1 \\
Carcinoma escamocelular & 17 & 9,2 & 14 & 7,6 \\
Carcinoma basocelular & 83 & 44,9 & 59 & 31,9 \\
Nevus atípico & 32 & 17,3 & 15 & 8,1 \\
Queratosis actínica & 16 & 8,6 & 26 & 14,1 \\
Otros nevus & 7 & 3,8 & 34 & 18,4 \\
Lesiones no malignas & 7 & 3,8 & 34 & 18,4 \\
\hline \hline
\end{tabular}

Tabla 5. Diagnósticos clínicos e histológicos en el grupo de pacientes con sospecha de lesiones premalignas y malignas a quienes se les practicó biopsia de piel. la sensibilidad del diagnóstico clínico y el valor pronóstico positivo.

Cuatro de los pacientes con diagnóstico comprobado de nevus atípico (26,7\%), tenían antecedentes familiares de melanoma maligno pero ninguno tenía antecedentes personales. En cuanto al antecedente personal de lesiones malignas o premalignas, dos de los pacientes con queratosis actínicas habían tenido carcinoma escamocelular. Uno de los dos pacientes con melanoma maligno, 42,9\% de los que tenía carcinoma escamocelular, $25,9 \%$ de los que tenía carcinoma basocelular y 19,2 \% con queratosis actínica, relataban antecedente de carcinoma basocelular.

Se revisaron los fototipos de las personas según el tipo histológico de lesión y se encontró que seguían predominando los fototipos 11 y 111.

Estudiando la exposición a radiación ultravioleta de los pacientes con diagnóstico comprobado de lesión premaligna o maligna, para la variable "Se quemó al sol antes de los 18 años”, se pudo observar que la alteración más frecuente fue carcinoma escamocelular $(64,3 \%)$ y que la mitad de las personas con melanoma maligno, carcinoma escamocelular y carcinoma basocelular practicaba actividades al sol. Los dos pacientes con melanoma maligno, el $42 \%$ de aquéllos a quienes se le comprobó carcinoma escamocelular y carcinoma basocelular, y el $46 \%$ de los que tenían queratosis actínica, trabajaban o habían trabajado al aire libre. Los dos pacientes con melanoma maligno y el $71,4 \%$ de los que presentaban carcinoma escamocelular, habían vivido en el campo. El 26,7 \% de los que tenían nevus atípicos habían ido a cámaras bronceadoras, frente a $11 \%$ de personas con carcinoma basocelular y queratosis actínicas, el 7,5\% con carcinoma escamocelular y ninguno de los pacientes con melanoma maligno. La presencia de lentigos solares se detectó en los dos pacientes con melanoma maligno, el 78,6 \% con carcinoma escamoce- lular, el $76,9 \%$ con queratosis actínicas y el $62,7 \%$ con carcinoma basocelular.

La presencia de nevus de unión, lentigos solares y efélides, se reportó en los dos pacientes con melanoma maligno y, en uno de ellos, un nevus atípico.

\section{Discusión}

Los programas de tamización de cáncer de piel se iniciaron como The Queensland Melanoma Project en Australia en los años 8o. En Estados Unidos surgieron como un programa educacional anual para la detección de melanoma maligno y cáncer de piel no melanoma, organizado por la American Academy of Dermatology en 1995. En el año 2000, la European Academy of Dermatology instauró una campaña anual de detección precoz de melanoma maligno denominada Euromelanoma Day.

El objetivo de estas jornadas ha sido disminuir la incidencia de cáncer de piel, mediante su detección temprana y el cambio de comportamiento de la población frente a la exposición a radiación ultravioleta, y como consecuencia de lo anterior, impactar en la morbilidad y mortalidad del cáncer cutáneo $3,8,9,10,20$.

Nuestro país, situado en el trópico, tiene un índice de radiación ultravioleta muy alto ${ }^{19} \mathrm{y}$ una alta frecuencia de cáncer de piel; aunque tenemos estadísticas limitadas, se ha reportado que es una de las causas más frecuentes de consulta ${ }^{21,22}$, y en la práctica dermatológica se diagnostica casi a diario. En Medellín se han hecho previamente dos estudios de detección temprana de cáncer de piel. El primero se llevó a cabo en todos los puntos de atención de una Entidad Promotora de Salud (EPS) de Antioquia. y se examinaron 407 adultos en mayo y junio del 2000. Se encontró una incidencia de lesiones premalignas y malignas del $8,8 \%{ }^{23}$. El segundo fue realizado en los dos centros de atención dermatológica de 


\begin{tabular}{|c|c|c|c|c|}
\hline \multirow{2}{*}{ Diagnóstico } & \multirow{2}{*}{ Diagnóstico clínico } & \multicolumn{2}{|c|}{ Diagnóstico histológico } & \multirow{2}{*}{ Total } \\
\hline & & Sí & No & \\
\hline \multirow{3}{*}{ Melanoma maligno } & Sí & 2 & 19 & 21 \\
\hline & No & 0 & 164 & 164 \\
\hline & Total & 2 & 183 & 185 \\
\hline \multicolumn{5}{|l|}{ Sensibilidad de $100 \%$} \\
\hline \multicolumn{5}{|l|}{ Valor pronóstico positivo de $9,5 \%$} \\
\hline \multirow{3}{*}{ Carcinoma escamocelular } & Sí & 6 & 11 & 17 \\
\hline & No & 8 & 160 & 168 \\
\hline & Total & 14 & 171 & 185 \\
\hline \multicolumn{5}{|l|}{ Sensibilidad de 42,9\% } \\
\hline \multicolumn{5}{|l|}{ Valor pronóstico positivo de $35,5 \%$} \\
\hline \multirow{3}{*}{ Carcinoma basocelular } & Sí & 51 & 32 & 83 \\
\hline & No & 8 & 94 & 102 \\
\hline & Total & 59 & 126 & 185 \\
\hline \multicolumn{5}{|l|}{ Sensibilidad de $86,4 \%$} \\
\hline \multicolumn{5}{|l|}{ Valor pronóstico positivo de $61,4 \%$} \\
\hline \multirow{3}{*}{ Queratosis actínica } & Sí & 11 & 5 & 16 \\
\hline & No & 15 & 154 & 169 \\
\hline & Total & 26 & 159 & 185 \\
\hline \multicolumn{5}{|c|}{$\begin{array}{l}\text { Sensibilidad de } 42,3 \% \\
\text { Valor pronóstico positivo de } 68,8 \%\end{array}$} \\
\hline \multirow{3}{*}{ Nevus atípico } & Sí & 11 & 21 & 32 \\
\hline & No & 4 & 149 & 153 \\
\hline & Total & 15 & 170 & 185 \\
\hline \multirow{2}{*}{\multicolumn{5}{|c|}{$\begin{array}{l}\text { Sensibilidad de } 73,3 \% \\
\text { Valor pronóstico positivo de } 34,4 \%\end{array}$}} \\
\hline & & & & \\
\hline \multirow{3}{*}{ Otros nevus } & Sí & 6 & 28 & 34 \\
\hline & No & 1 & 150 & 151 \\
\hline & Total & 7 & 178 & 185 \\
\hline \multirow{2}{*}{\multicolumn{5}{|c|}{$\begin{array}{l}\text { Sensibilidad de } 85,7 \% \\
\text { Valor pronóstico positivo de } 17,6 \%\end{array}$}} \\
\hline & & & & \\
\hline \multirow{3}{*}{ Otros diagnósticos no malignos } & Sí & 7 & 0 & 7 \\
\hline & No & 27 & 151 & 178 \\
\hline & Total & 34 & 151 & 185 \\
\hline \multicolumn{5}{|l|}{ Sensibilidad de $20,6 \%$} \\
\hline Valor pronóstico positivo de 100 & & & & \\
\hline
\end{tabular}

Tabla 6. Sensibilidad del diagnóstico clínico comparado con el resultado de dermatopatología y valor pronóstico positivo de la prueba.

la Universidad CES, en mayo de 2005, y se evaluaron 736 personas mayores de 18 años, de las cuales, $10 \%$ tuvo sospecha clínica de lesiones malignas, $13,7 \%$ de lesiones premalignas y $19,7 \%$ presentaba algún tipo de alteración producida o agravada por el sol$^{24}$.

Asocolderma, consciente de la necesidad de llegar a la población afectada, planea realizar una jornada de detección precoz de cáncer de piel a nivel nacional, en mayo de 2012; como parte de la estrategia inicial, se llevó a cabo una jornada piloto en Medellín los días 17, 18 y 19 de mayo de 2011, cuyos resultados se presentan en este artículo.

En esta jornada participaron en forma voluntaria 64 dermatólogos y residentes de Dermatología, lo que equivale a 43,2 \% de estos profesionales de Medellín. Esta participación se considera muy importante, teniendo en cuenta que era la primera jornada, lo que revela la excelente disposición para participar en eventos de pro- 


\title{
"Aunque no existen estudios de población de los fototipos de piel en Colombia, en los
}

\author{
estudios previos en la ciudad se demuestra un \\ claro predominio de los fototipos II y III que en \\ la población estudiada, sumaban el 75,8 \%”.
}

yección a la comunidad por parte de los dermatólogos de Medellín.

Se encuestaron 2.404 personas, de las cuales, se atendió el 52,62 \% que, cumpliendo los criterios de inclusión, pudieron ser evaluadas por el dermatólogo en las citas disponibles, de acuerdo con el número de horas donadas por ellos. La convocatoria de la campaña fue bien aceptada por la población y por los dermatólogos; sin embargo, la demanda superó la disponibilidad de citas y se quedaron pacientes sin atender.

De los encuestados telefónicamente, el 67,6 \% eran mujeres, con edades entre los 18 y los 90 años (promedio, 48 años), y el $80 \%$ tenía algún seguro de salud. La mayor frecuencia de mujeres en la jornada podría reflejar que ellas están más dispuestas al cuidado de la salud. Llama la atención que, aunque la afiliación al sistema de seguridad social es obligatoria, el $20 \%$ de los pacientes no tenía ningún tipo de seguro de salud y, en general, la mayoría de los pacientes no habían sido atendidos por la sospecha de cáncer de piel. Las dificultades para el acceso a los servicios de salud también se presentan en países desarrollados, como los Estados Unidos. En la evaluación de los primeros 15 años de las jornadas de cáncer de piel de la American Academy of Dermatology, de 1985 a 1999, el $8 \%$ de las personas encuestadas no tenía seguro social, el $80 \%$ no iba regularmente al dermatólogo y al 6o \% nunca se le había examinado su piel por parte de un médico ${ }^{25}$. Además, el $61 \%$ eran mujeres con una edad promedio de 52 años, semejante a lo reportado en la jornada de Medellín.

En la entrevista telefónica, la mitad dijo que habían tenido insolaciones en la infancia y ese mismo porcentaje no usaba protectores antisolares. Una tercera parte trabajaba al aire libre y dos terceras partes $(69,9 \%)$ se quemaban con facilidad. El motivo de consulta más frecuente fue "manchas, pecas o lunares" (72,6 \%); todas estas lesiones están relacionadas con la exposición solar. Respecto a la aparición de cambios en piel, 68,1\% de los pacientes manifestó que les salían "lunares" (nevus) regularmente, más de la mitad (57,3\%) eran de aparición reciente y el $62,7 \%$ eran de diferentes tonos de café. El 31,2 \% refería historia personal o familiar de cáncer de piel. El cambio reciente de los nevus, los diferentes tonos de café y la historia personal o familiar de cáncer de piel, son factores de riesgo importantes para la aparición de melanoma maligno ${ }^{26}$.

En la jornada de Medellín, de los 1.265 pacientes que fueron citados a evaluación por el dermatólogo al cumplir los criterios de inclusión, 246 (19,4\%) tuvieron lesiones de aspecto premaligno o maligno, según el criterio clínico del dermatólogo, y 149 personas $(11,7 \%)$ ameritaron la toma de biopsia. Esto contrasta con los resultados de los primeros 15 años del programa de la American Academy of Dermatology, en los que se hizo diagnóstico presuntivo de cáncer o lesiones premalignas en $30 \%$ de la población evaluada ${ }^{25}$.

$\mathrm{Al}$ analizar los datos de la encuesta diligenciada por el dermatólogo, solo un promedio de $80 \%$ de los datos estaba completo; la falta de un instructivo para el diligenciamiento de la historia, fue una de las posibles razones. Para la jornada nacional del 2012, es muy importante motivar a los médicos participantes para el diligenciamiento completo del formulario y adjuntar un instructivo.

Los datos sobre exposición a radiación ultravioleta de las 246 personas con sospecha clínica de lesiones 
premalignas o malignas examinadas por el dermatólogo, fueron muy semejantes a los de los encuestados por teléfono, y muestran una alta exposición solar, insolaciones antes de los 18 años y falta de medidas preventivas como el uso del protector antisolar en la mitad de la población. En la población estudiada, el uso de cámaras bronceadoras corresponde a $12 \%$; en este estudio, su uso fue más frecuente en los pacientes con nevus atípicos.

Aunque no existen estudios de población de los fototipos de piel en Colombia, en los estudios previos en la ciudad se demuestra un claro predominio de los fototipos II y III ${ }^{23,24}$ que en la población estudiada, sumaban el 75,8 \%. La baja habilidad para broncearse sumada a la alta exposición solar y al poco uso del protector antisolar, son factores de riesgo importantes para la aparición de cáncer de piel. En la población estudiada en los Estados Unidos predominaron los fototipos I y II, pero en las jornadas mediterráneas del Euromelanoma Day, en Grecia, $76 \%$ eran de fototipos II y III, $47 \%$ había sufrido quemaduras solares en la niñez, $5 \%$ tenía historia personal o familiar de melanoma maligno, $12 \%$ trabajaba al aire libre, $40 \%$ realizaba actividades al sol, 2,9 \% asistía a sesiones de cámaras bronceadoras, $44 \%$ tenía lentigos solares al examen clínico y 31,2 \% presentaba nevus displásicos ${ }^{27}$.

La presencia de nevus de unión, el número de nevus presentes en el cuerpo y nevus atípicos, las altas dosis de radiación ultravioleta recibidas y los antecedentes personales y familiares de cáncer de piel, son factores de riesgo para la aparición de melanoma ${ }^{3,28}$. En este análisis, cuatro pacientes con nevus atípicos tenían historia familiar (pero no personal) de melanoma maligno y uno de los dos pacientes con melanoma maligno tenía nevus atípico. Dos terceras partes de los examinados tenían nevus de unión, la mayoría con recuentos inferiores a 25 , pero una cuarta parte tenía de 25 a 100 lesiones y $37,7 \%$ eran clínicamente atípicos. En el examen físico se corroboró la alta exposición solar descrita en el interrogatorio, con la presencia de efélides ( $49 \%$ ) y lentigos solares $(75 \%)$. Al preguntar sobre antecedentes, 12 tenían algún familiar con melanoma maligno, 1 había tenido melanoma maligno; 5 , carcinoma escamocelular; 30, carcinoma basocelular, y 5, otro cáncer cutáneo.

De las 1.265 personas que cumplieron los criterios de inclusión y fueron citadas a la campaña, hubo sospecha clínica de melanoma maligno en 21 (1,66\%), de carcinoma escamocelular en 17 (1,34\%), de carcinoma basocelular en $83(6,56 \%)$, de queratosis actínicas en 71 $(5,6 \%)$ y de nevus atípicos en 71 (5,6\%). En el estudio de los primeros 15 años de las jornadas de cáncer de piel de la American Academy of Dermatology, los diagnósticos presuntivos fueron: melanoma maligno (o,8 \%), carcinoma escamocelular (1\%), carcinoma basocelular $(9 \%)$, queratosis actínicas $(23 \%)$ y nevus atípico $(9 \%)^{25}$.

A 149 pacientes con sospecha clínica de lesiones premalignas o malignas, se les tomaron 185 biopsias de piel. Por estudio histopatológico, se comprobaron 75 lesiones malignas y 41 premalignas, lo que representa $5,9 \%$ y $3,2 \%$, respectivamente, de los que se citaron para evaluación. Los siguientes fueron los diagnósticos histológicos: melanoma maligno, 2 (o,16 \%); carcinoma basocelular, 59 (4,66\%); carcinoma escamocelular, 14 $(1,1 \%)$; queratosis actínicas, 26 (2 \%), y nevus atípicos, 15 (1,2 \%); los porcentajes se calcularon sobre el total de población citada a la campaña. En la campaña de Euromelanoma Day de Grecia del 2003 al 2004, se comprobó el diagnóstico de cáncer de piel no melanoma en 6,4 \% de la población estudiada, distribuidos así: melanoma maligno, 0,35 \%; carcinoma escamocelular, $1 \%$, y carcinoma basocelular, 5,4\% ${ }^{27}$.

La utilidad de las campañas de detección de cáncer de piel se ha demostrado en diferentes poblaciones del mundo. En Alemania, por ejemplo, desde el 2003 la Society of Dermatological Prevention implementó el proyecto SCREEN (Skin Cancer Research to Provide Evidence for Effectiveness of Screening in Northern Germany).

Este es el programa de detección sistemática de cáncer de piel más grande del mundo. Consiste en dos pasos de detección del melanoma maligno y del cáncer de piel no melanoma. El primer paso fue llevado a cabo por personal sin formación como dermatólogo, que recibió un entrenamiento previo, y el segundo paso fue la evaluación por el dermatólogo para los que tenían lesiones sospechosas. Algunos pacientes tuvieron acceso directo al dermatólogo.

De los 1,88 millones de ciudadanos elegibles, participaron 360.288 (19\% de la población). Se evaluó toda la piel y se tomaron biopsias de las lesiones sospechosas. Se encontraron 3.103 tumores malignos de la piel (2.911 pacientes, 0,8 \% de los pacientes reclutados en el estudio); entre estos, se presentó melanoma maligno en $585(0,16 \%)$, carcinoma basocelular en 1.961 (o,54 \%), y carcinoma escamocelular en 392 (o,1 \%). La incidencia de melanoma invasivo aumentó $34 \%$ durante el año del proyecto SCREEN. Cinco años después, se presentó disminución de la mortalidad por melanoma (hombres: observado 0,79 por 100.000 y esperado 2 por 100.000 ; mujeres: observado 0,66 por 100.000 y esperado 1,3 por 100.000) y de la gravedad de los casos detectados.

El proyecto demostró que la detección sistemática del cáncer de piel a gran escala es factible y puede reducir la mortalidad. Con base en estos resultados, se instauró en Alemania un programa nacional de detección temprana de cáncer de piel, en el 2008.

En comparación con lo referido en la literatura cientí- 
fica, en el proyecto SCREEN se detectó o,16 \% de melanoma maligno y, en la jornada de Medellín, de los 1.265 sujetos citados a evaluación, $2(0,15 \%)$ presentaron melanoma maligno. El porcentaje de carcinoma basocelular y carcinoma escamocelular fue cinco veces mayor en la jornada realizada en Medellín, que lo descrito en Alemania, así: en Medellín, carcinoma basocelular, 59 $(4,7 \%)$, y carcinoma escamocelular, 14 (1,1\%), frente a $0,54 \%$ y $0,1 \%$, respectivamente, en Alemania ${ }^{28}$.

La sensibilidad del examen visual hecho por el dermatólogo se ha comparado con otras pruebas diagnósticas, como sangre oculta para el cáncer de colon, mamografía para el cáncer de mama y Papanicolaou para el cáncer de cuello uterino ${ }^{29}$. En la jornada de Medellín, la sensibilidad del diagnóstico clínico de melanoma maligno fue de $100 \%$, 42,86 \% para carcinoma escamocelular, $86,44 \%$ para carcinoma basocelular, $42,31 \%$ para queratosis actínicas, y 73,3\% para nevus. En estudios previos ha sido $97 \%$ para melanoma maligno, $94 \%$ para carcinoma basocelular y $89 \%$ para carcinoma escamocelular, y la sensibilidad total fue de 93,3 \% ${ }^{29}$.

Los dos pacientes con diagnóstico comprobado de melanoma maligno eran mujeres, ambas tenían las lesiones en la cara, de uno y tres años de evolución; a esta última se le había practicado crioterapia previa con posterior reaparición de la lesión. El diagnóstico histopatológico fue lentigo maligno en las dos, se observaban en fase de crecimiento radial y estaban in situ (respetaban la membrana basal). A 21 pacientes se les sospechó clínicamente melanoma maligno pero solo en dos se comprobó por histopatología; esto sugiere que, ante lo devastador de este tipo de cáncer, el dermatólogo permanece alerta procurando un diagnóstico temprano. En las campañas del Euromelanoma Day de 2000, 2001 y 2002 en España, de 164 lesiones sospechosas de melanoma maligno, se comprobaron histológicamente 31 casos, con una correlación clínico-histológica de $23,3 \%{ }^{10}$.

El valor pronóstico positivo del diagnóstico clínico en las lesiones premalignas o malignas fue mayor en las queratosis actínicas $(68,8 \%)$, seguido por el del carcinoma basocelular $(61,4 \%)$, y el más bajo fue para melanoma maligno (9,5\%). Sin embargo, este último valor es semejante al obtenido en los estudios de la American Academy of Dermatology sobre melanoma maligno de 1985 a 2005, cuyo valor pronóstico positivo estuvo entre 6 y $17 \%$ 3. En Grecia, de 2003 a 2004, de 171 con sospecha clínica se comprobaron 19, con un valor pronóstico positivo de $11,4 \%{ }^{27}$.

En esta jornada piloto de detección de cáncer de piel, se dejó en libertad al dermatólogo para examinar toda la piel o solo la expuesta y la zona de la lesión referida por el paciente. El examen de toda la piel del cuerpo ha demostrado ser más útil y confiable. En un estudio de 14.381 pacientes, se detectaron 40 (0,3\%) con melanoma y 299 (2,1\%) con, por lo menos, un cáncer de piel no melanoma con este tipo de examen.

Los autores calculan que se debe practicar examen de toda la piel del cuerpo a 47 pacientes, para encontrar un cáncer de piel de cualquier tipo, y a 400 pacientes, para detectar un melanoma. El riesgo de no diagnosticar un tumor maligno si no se practica un examen de toda la piel del cuerpo, es de $2,17 \%$. Los factores que aumentan significativamente la oportunidad de encontrar un cáncer de piel, son: edad, sexo masculino, antecedentes de un cáncer de piel no melanoma, el tipo de piel blanca, un tumor de la piel como motivo de consulta y la presencia de lesiones en las áreas descubiertas ${ }^{30}$.Por lo tanto, la recomendación para la jornada nacional de 2012, es hacer un examen de toda la piel del cuerpo, lo que requiere un mayor tiempo de evaluación para cada paciente.

A pesar de las dificultades y limitaciones, este tipo de campaña tiene una gran importancia en la población colombiana que, aunque tiene una alta cobertura en salud, no tiene acceso al oportuno examen por un dermatólogo, no conoce los factores de riesgo ni las características de las lesiones sospechosas de cáncer de piel y está expuesta a una alta dosis de radiación ultravioleta sin la adecuada protección.

Además de la detección del cáncer de piel, esta jornada contribuyó, de forma no menos importante, a la educación de la población sobre los signos de alarma del melanoma maligno, por medio de volantes, afiches y medios masivos de comunicación.

A este esfuerzo educativo debe dársele continuidad y ser ampliado para otras lesiones, como las queratosis actínicas, el carcinoma basocelular y el carcinoma escamocelular. En la función del dermatólogo es primordial la educación al paciente que permita la promoción y prevención, y contribuir al diagnóstico oportuno del cáncer de piel.

\section{Agradecimientos}

A los dermatólogos, en su mayoría miembros de SADE y Asocolderma, que donaron su tiempo y participaron en la jornada, doctores: Verónica Molina, Pilar Navarro, Sandra Yepes, Lina María Arango, Paula Mejía, María Clemencia Gómez, José Johani García, Luis Fernando Gómez, Carlos Lozano, Andrés Anaya, María Adelaida Echeverry, Diego Espinosa, Isabel Arredondo, Mónica Zapata, Ana María Aristizábal, Elina Bedoya, Lina María Quiroz, Amparo Ochoa, Milton Mejía, Clara Marcela Jaramillo, Norma González, Juan Pedro Velásquez, Jorge E. Sánchez, Olga Castaño, Marcela González, Mónica 
Escobar, María Cristina Lotero, Mónica A. Gaviria, Jorge Luis Duque, María Salomé Ramírez, Mary Ann Robledo, Carolina Velásquez, Ángela Londoño, Beatriz Sierra, Ana Villa, Claudia Vélez, Liliana Tamayo, Gloria Velásquez, Carlos Montealegre, Andrea Vargas, Juan Carlos Wolff, Víctor Muñoz, Marta Sierra, Gabriel Ceballos, Juan Guillermo Hoyos, Isabel Vásquez, Margarita Velásquez y Ângela Zuluaga

A los residentes de Dermatología que participaron en la campaña: Cristina Escobar, Catalina Restrepo, Ana María Mejía, Diana Zuluaga, Yurledy Del Río, Alexander Usuga, Óscar Valencia, Freya Álvarez, Eugenia Castañeda, Pamela Gallego, Natalia De la Calle, Franchezca Zapata, Nathalie Morales, María Alejandra Zuluaga, María Ximena Tobón, Andrea Solórzano y Ángel Jaimes.

A las escuelas de Dermatología de la Universidad CES y la Universidad de Antioquia.

A los laboratorios de Dermatopatología de la Universidad de Antioquia y la Universidad CES, y a los profesores de Dermatopatología, Luis Alfonso Correa y Ana Cristina Ruiz.

A Diana Correa, estudiante de Medicina de la Universidad de Antioquia, por el apoyo en el manejo de las bases de datos y encuestas.

A Bibiana Castro, epidemióloga del CES, por su invaluable apoyo para el análisis de los resultados.

A los Laboratorios Percos, por la financiación y el apoyo logístico.

\section{Referencias}

1. LeBlanc WG, Vidal L, Kirsner RS, Lee DJ, Caban-Martínez AJ, McCollister KE, et al. Reported skin cancer screening of US adult workers. J Am Acad Dermatol. 2008;59:55-63.

2. Saraiya M, Glanz K, Briss PA, Nichols P, White C, Das D, et al. Interventions to prevent skin cancer by reducing exposure to ultraviolet radiation: A systematic review. Am J Prev Med. 2004;27:422-66.

3. Goldberg MS, Doucette JT, Lim HW, Spencer J, Carucci JA, Rigel DS. Risk factors for presumptive melanoma in skin cancer screening: American Academy of Dermatology National Melanoma/ skin cancer screening program experience 2001-2005. J Am Acad Dermatol. 2007;57:60-6.

4. Geller AC, Swetter SM, Oliveria S, Dusza S, Halpern AC. Reducing mortality in individuals at high risk for advanced melanoma through education and screening. J Am Acad Dermatol. 2011;65:S87-94.

5. Demierre MF. Thin melanomas and regression, thick melanomas and older men: Prognostic implications and perspectives on secondary prevention. Arch Dermatol. 2002;138:678-82.

6. Hypolito Silva J, Costa Soares de Sa B, Ribeiro de Avila AL, Landman G, Duprat Neto JP. Atypical mole syndrome and dys- plastic nevi: Identification of populations at risk for developing melanoma -review article. Clinics. 2011;66:493-9.

7. Miller DR, Geller AC, Wyatt SW. Melanoma awareness and selfexamination practices: Results of a United States survey. J Am Acad Dermatol. 1996;34:962-7.

8. Smith T. The Queensland Melanoma Project: An exercise in health education. BMJ. 1979;1:253-4.

9. Koh HK, Norton LA, Geller AC, Sun T, Rigel DS, Miller DR, et al. Evaluation of the AAD National Skin Cancer Early Detection and screening program. Part I. J Am Acad Dermatol. 1996;34:971-8.

10. Conejo-Mir J, Bravo J, Díaz-Pérez JL, Fernández-Herrera J, Guillén C, Martí R, et al. Día del Euromelanoma. Resultados en España de las campañas de 2000, 2001 y 2002. Actas Dermosifiliogr. 2005;96:217-21.

11. Qureshi AA, Wei-Passanese EX, Li T, Han J. Host risk factors for the development of multiple non-melanoma skin cancers. J Eur Acad Dermatol Venereol. 2012, Feb 24. doi: 10.1111/j.14683083.2012.04483.x.

12. Bozikov K, Taggart I. Metastatic basal cell carcinoma: Is in filtrative/morpheaform subtype a risk factor? Eur I Dermatol. 2006;16:691-2.

13. Dal H, Boldemann C, Lindelöf B. Trends during a half century in relative squamous cell carcinoma distribution by body site in the Swedish population: Support for accumulated sun exposure as the main risk factor. J Dermatol. 2008;35:55-62.

14. Benjamin C, Ananthaswamy HN. p53 and the pathogenesis of skin cancer. Toxicol Appl Pharmacol. 2007;224:241-8.

15. Bouwes JN, Plasmeijer EI, Feltkamp MC. B papillomavirus infection and skin cancer. J Invest Dermatol. 2008;128:1355-8.

16. Bonerandi JJ, Beauvillain C, Caquant L, Chassagne JF, Chaussade $\mathrm{V}$, Clavère $\mathrm{P}$, et al. Guidelines for the diagnosis and treatment of cutaneous squamous cell carcinoma and precursor lesions. J Eur Acad Dermatol Venereol. 2011;25(Suppl.5):1-51.

17. Feldman SR, Fleischer AB Jr. Progression of actinic keratosis to squamous cell carcinoma revisited: Clinical and treatment implications. Cutis. 2011;87:201-7.

18. Yanofsky VR, Mercer SE, Phelps RG .Histopathological variants of cutaneous squamous cell carcinoma: A review. J Skin Cancer. 2011;210:8-13.

19. Sanclemente G, Hernández G. Altos índices de radiación ultravioleta en Medellín y en una localidad del oriente antioqueño (Colombia). Iatreia. 2010;23:119-23.

20. Scheneider JS, Moore DH, Mendelsohn ML. Screening program reduced melanoma mortality at the Lawrence Livermore National laboratory, 1984 to 1996. J Am Acad Dermatol. 2008;58:741-9.

21. Sanclemente G, Mahecha M, Guzmán C. Enfermedades de la piel más frecuentes en la consulta dermatológica del Hospital Universitario San Vicente de Paúl y del Hospital Infantil, Medellín, Colombia 1999. Acta Méd Col. 2001;26:240-4.

22. Sánchez G, Nova J, Arias N. Prácticas frente a la radiación ultravioleta y características epidemiológicas de un grupo de pacientes con carcinoma basocelular en un centro de referencia nacional en Colombia. Rev Colomb Cancerol. 2010;14:144-51.

23. González M, Lotero MC, Quiroz L, Navarro M del P, Jaramillo CM, Gaviria MB, et al. Conocimientos y prácticas frente a la exposición solar y tamizaje de cáncer de piel en usuarios de una institución prestadora de salud de Antioquia mayo-junio 2000. Rev Asoc Colomb Dermatol. 2001;9:487-95.

24. Restrepo JC, Zuluaga A, Ochoa FL, Jiménez SB, Castaño OL, Uribe 
C et al. Jornada de prevención y detección de cáncer de piel en personas mayores de 18 años. Medellín, mayo de 2005. Universidad CES. Rev CES Med. 2009;23:93-103.

25. Geller AC, Zhang Z, Sober AJ, Halpern AC, Weinstock MA, Daniels $\mathrm{S}$, et al. The first 15 years of the American Academy of Dermatology Skin Cancer Screening Programs: 1985-1999. J Am Acad Dermatol. 2003;48:34-41.

26. Geller AC, Swetter SM, Brooks K, Demierre MF, Yaroch AL. Screening, early detection, and trends for melanoma: Current status (2000-2006) and future directions. J Am Acad Dermatol. 2007;57:555-72.

27. Stratigos A, Nicolaou V, Kedicoglou S, Antoniou C, Stefapaki I, Hoidemenos G, et al. Melanoma/skin cancer screening in Mediterranean country: Results of the Euromelanoma screening day campaign in Greece. J Eur Acad Dermatol Venereol. 2007;21:56-62.
28. Breitbart EW, Waldmann A, Nolte S, Capellaro M, Greinert R, Volkmer B, et al. Systematic skin cancer screening in Northern Germany. J Am Acad Dermatol. 2012;66:201-11.

29. Rampen FH, Casporie-van Velsen JA, van Huystee BE, Kiemeney LA, Schouten LJ. False negative findings in skin cancer and melanoma screening. J Am Acad Dermatol. 1995;33:59-63.

30. Argenziano G, Zalaudek I, Hofmann-Wellenhof R, Bakos RM, Bergman W, Blum A, et al. Total body skin examination for skin cancer screening in patients with focused symptoms. J Am Acad Dermatol. 2012;66:212-9. 